\title{
Iterative Blind Channel Estimation for OFDM Receivers
}

\author{
Karl-Dirk Kammeyer, Thorsten Petermann, and Sven Vogeler \\ University of Bremen, Department of Communications Engineering \\ P.O. Box 3304 40, D-28334 Bremen, Germany \\ E-mail: $\{$ kammeyer, petermann, vogeler\}@comm.uni-bremen.de
}

\begin{abstract}
Finite-Alphabet based blind channel estimation in OFDM systems is known to be extremely complex due to an exhaustive search to be performed over a tremendous number of channel coefficient combinations. In this paper, we present a novel blind channel estimator, which dramatically reduces this number of coefficient combinations to be checked without a significant deterioration in estimation quality. Hence, the new low complexity approach enables the application of blind channel estimators based on the finite alphabet set even if the transmitted data are high-rate modulated. Furthermore, we show that the performance of blind channel estimation can be improved by an iterative process based upon the capabilities of channel coding. Using bit error rates (BERs) before and after channel decoding, the algorithm is tested with simulations and compared to other blind and non-blind channel estimators.
\end{abstract}

\section{Introduction}

In recent years, Orthogonal Frequency Division Multiplexing (OFDM) has become one of the most important techniques for high-rate wireless data transmission. Especially, it has been proposed for the European HIPERLAN/2 standard and the American equivalent IEEE802.11a, which are two similar concepts for broadband wireless local area networks (WLAN) in the $5 \mathrm{GHz}$ band. Furthermore, OFDM is currently being adopted and tested to digital audio and video broadcasting (DAB/DVB) and high speed asynchronous digital subscriber line (A-DSL) modems.

Since most of the mentioned standards include coherent data demodulation, the transmission channel has to be estimated. Generally, this is achieved by non-blind channel estimators exploiting additionally transmitted training data. Moreover, the training sequences have to be transmitted periodically, since the channel in wireless applications

${ }^{*}$ This research was supported by the German NSF (DFG contr. \#Ka 841/5-1). normally is time variant. In order to increase bandwidth efficiency, blind channel estimation, on the other hand, is well motivated since it avoids the need of any training data. In recent publications, several blind channel estimation approaches based on cyclostationarity $[2,3]$ or subspace decompositions [4] have been tested for OFDM systems. Both classes of estimators are known to require long enough data records, in order to derive unbiased channel estimates. While subspace methods in general suffer from fading subcarriers (i.e. the channel has nulls on some subcarriers), the main drawback of algorithms based on cyclostationary statistics is their sensibility towards "singular" channel classes with common subsystems (i.e. common zeros) in all polyphase subchannels. Furthermore, cyclostationary methods require some excess bandwidth. In [6], a novel blind channel estimator has been presented which is based on the knowledge that the modulated and transmitted data are confined to a finite alphabet set. This so-called Minimum Distance (MD) algorithm copes with all the problems mentioned before. In this paper, we present the new Minimum Impulse Length (MIL) approach, which shows a better estimation performance than MD. However, both MIL and $\mathrm{MD}$ require an enormous computional effort. In contrast, our Clustered SubCarriers (CSC) scheme dramatically reduces this effort without any significant deterioration in estimation quality. Furthermore, estimation quality can be improved by an iterative channel estimation scheme even if the channel is highly time variant.

The paper is organized as follows: Section 2 gives an overview of the OFDM system. In section 3, the principles of non-blind and blind channel estimation are described. Exploiting the capabilities of channel coding, we present in section 4 the Turbo Channel Estimation for an initial nonblind or blind estimator. After showing some simulation results in section 5, the paper is concluded in section 6 .

\section{Data Transmission in OFDM systems}

Figure 1 shows the conventional OFDM system with Cyclic Prefix (CP). The CP of length $N_{c p}$ larger than the 
channel order $q$ avoids the received data from being disturbed by inter-symbol (ISI) or inter-carrier interference (ICI). In the transmitter, the channel encoded information

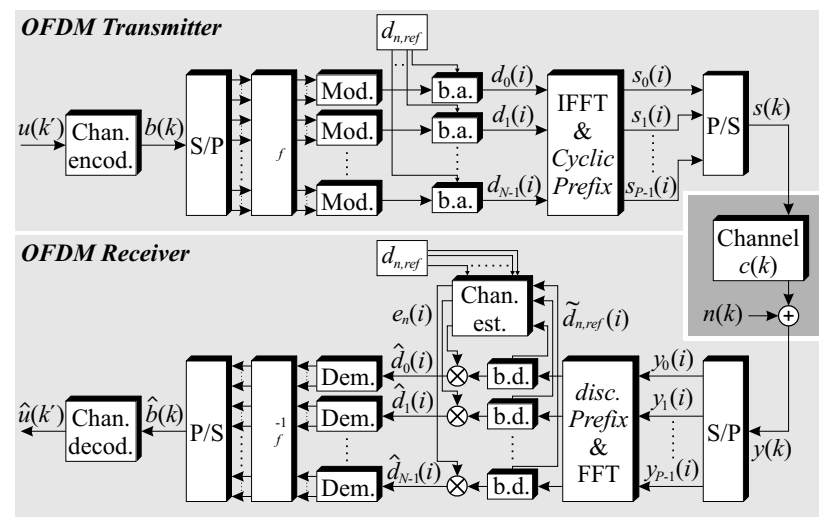

Figure 1: Conventional OFDM system with Cyclic Prefix

stream $b(k)$ is serial-to-parallel converted $(\mathrm{S} / \mathrm{P})$, interleaved $\left(\Pi_{f}\right)$, modulated, and assembled into so-called OFDM symbols $\mathbf{d}(i):=\left[d_{0}(i), d_{1}(i), \ldots, d_{N-1}(i)\right]^{T}$ of length $N$.

After P/S conversion, the OFDM sequence ${ }^{1} s(k)$ is transmitted over the time discrete channel $c(k)=\left(g_{c} *\right.$ $\left.c_{c} * h_{c}\right)\left.(t)\right|_{t=k T}$, where $*$ denotes convolution, $T$ is the chip period and $g_{c}(t), h_{c}(t)$, and $c_{c}(t)$ are the time continuous transmit and receive filters and the physical channel, respectively. The received and sampled signal is given by

$$
y(k)=\sum_{l=0}^{q} c(l) \cdot s(k-l)+n(k),
$$

where $n(k)$ is Additive Gaussian Noise (AGN) colored by the receive filter $h_{c}(t)$. Under the assumption that ICI and ISI have been prevented from occurring $\left(N_{c p}>q\right)$, the $i$ th received OFDM symbol $\tilde{\mathbf{d}}(i)$ after $\mathrm{S} / \mathrm{P}$ conversion is calculated by

$$
\tilde{\mathbf{d}}(i)=\mathbf{D}_{C} \mathbf{d}(i)+\tilde{\mathbf{n}}(i),
$$

where $\mathbf{D}_{C}:=\operatorname{diag}\left[C\left(e^{j 0}\right), C\left(e^{j \frac{2 \pi}{N}}\right), \ldots, C\left(e^{j \frac{2 \pi}{N}(N-1)}\right)\right]$ is a diagonal matrix with diagonal elements $C\left(z_{n}\right):=$ $\sum_{l=0}^{q} c(l) z_{n}^{-l}$ evaluated at the subcarriers $z_{n}=e^{j \frac{2 \pi}{N} n}$ for each $n \in[0, N-1]$. From (2) it is obvious that the channel influence is reduced to one complex Rayleigh fading factor (channel coefficient) on each subcarrier.

Since each OFDM symbol has to be demodulated coherently, the channel coefficients $C\left(\rho_{n}\right):=C\left(e^{j \frac{2 \pi}{N} n}\right)$ have to be estimated. Therefore, let $\hat{C}\left(\rho_{n}\right)$ denote the estimate of each subcarrier $n$ which will be used to equalize $\tilde{d}_{n}(i)$

$$
\hat{\mathbf{d}}(i)=\mathbf{D}_{e} \tilde{\mathbf{d}}(i),
$$

\footnotetext{
${ }^{1} k=i N+n, \quad n \in[0, N-1] \quad$ defines the chip index, where $n$ is the subcarrier index in frequency domain and $i$ characterizes the OFDM symbol index in time domain.
}

where $\mathbf{D}_{e}=\operatorname{diag}\left[e_{0}(i), e_{1}(i), \ldots, e_{N-1}(i)\right]$ with equalizer coefficients $e_{n}(i)=1 / \hat{C}\left(\rho_{n}\right)$. Finally, each OFDM symbol is de-interleaved $\left(\Pi_{f}^{-1}\right), \mathrm{P} / \mathrm{S}$ converted, and channel decoded into bits $\hat{u}\left(k^{\prime}\right)$. If channel decoding is based on soft values, it is important that the demodulated bits of each subcarrier $n$ are multiplied with the channel state information $\left|\hat{C}\left(\rho_{n}\right)\right|^{2}$ before de-interleaving (not shown in Fig. 1).

\section{OFDM Channel Estimation}

According to the PHY layer of HIPERLAN/2 and IEEE802.11a, several OFDM symbols are combined to bursts of different lengths. In case of non-blind channel estimation, each burst is preceded by a preamble consisting of two identical training symbols $d_{n, r e f}$ (block burst assembly - b.a. in Fig. 1). Section 3.1 will give a short overview of a non-blind estimator. If, on the other hand, the channel shall be estimated blindly, a burst only contains information-bearing symbols increasing the bandwidth efficiency. Introductorily, we already listed the drawbacks and advantages of blind estimation algorithms of different classes. In section 3.2, we will present two new FiniteAlphabet based blind channel estimators.

\subsection{Non-Blind Channel Estimation}

According to section 2, only one coefficient per subcarrier has to be estimated. With the given burst structure, however, the reference based channel estimation after burst disassembly (b.d., see Fig. 1) delivers two subcarrier coefficients at the beginning of each burst

$$
\hat{C}\left(\rho_{n}, i\right)=\frac{\tilde{d}_{n, r e f}(i)}{d_{n, r e f}} \quad \text { with } \quad i \in\{0,1\} .
$$

If the channel is assumed to be quasi time invariant over one burst period, the averaged coefficients

$$
\hat{C}\left(\rho_{n}\right)=\frac{1}{2} \cdot\left(\hat{C}\left(\rho_{n}, 0\right)+\hat{C}\left(\rho_{n}, 1\right)\right)
$$

can be applied to the equalization of all OFDM symbols belonging to that burst.

In general, the estimated channel transfer coefficient (5) is disturbed by AGN. By exploiting the correlations between adjacent subcarrier coefficients and taking into account that the channel impulse response does not violate the cyclic prefix $\left(N_{c p}>q\right)$, a noise reduction with

$$
\hat{c}(k)=\left\{\begin{array}{ll}
\hat{c}(k) & 0 \leq k \leq N_{c p}-1 \\
0 & N_{c p} \leq k \leq N-1
\end{array},\right.
$$

can further improve estimation quality [5]. 


\subsection{Blind Channel Estimation}

In [6], Zhou and Giannakis have presented the so-called Minimum Distance (MD) algorithm and its complexity reduced version MMD (Modified Minimum Distance). Both MD and MMD are based on the knowledge that the $M$-ary modulated signals ${ }^{2}$ are drawn from a finite alphabet set of size $M$, i.e. $d_{n}(i) \in\left\{\zeta_{m}\right\}_{m=1}^{M}$. From this it is easy to see that

$$
\begin{aligned}
& \prod_{m=1}^{M}\left[d_{n}(i)-\zeta_{m}\right]= \\
& \quad d_{n}^{M}(i)+\alpha_{1} d_{n}^{M-1}(i)+\cdots+\alpha_{M} \stackrel{!}{=} 0,
\end{aligned}
$$

where $\alpha_{1}, \ldots, \alpha_{M}$ are determined by the constellation points $\left\{\zeta_{m}\right\}_{m=1}^{M}$. With $d_{n}(i) \neq 0$, let $J$ denote the smallest index for which $\alpha_{J} \neq 0 ; \alpha_{m}=0, \forall m \leq J$. The calculation of (7) shows that $J=M$ for $M$-ary PSK and $J=4$ for $M$ ary QAM with $M=16,32,64,128$, and 256 . Concerning the algorithms' complexity, the fact that $J \leq M$ (and for large signal constellations $J \ll M$ ) plays a very important role.

In [7], it was proven that for any signal constellation $\mathrm{E}\left\{d_{n}^{J}(i)\right\}=-(J / M) \alpha_{J} \neq 0$, where $\mathrm{E}\{\cdot\}$ denotes expectation value. With $\mathrm{E}\left\{\tilde{d}_{n}^{J}(i)\right\}=C^{J}\left(\rho_{n}\right) \mathrm{E}\left\{d_{n}^{J}(i)\right\}$ and the replacement of $\mathrm{E}\left\{d_{n}^{J}(i)\right\}$ by consistent sample averages (over $I$ OFDM symbols), $C^{J}\left(\rho_{n}\right)$ is estimated as:

$$
\hat{C}^{J}\left(\rho_{n}\right)=\frac{-M}{J \alpha_{J}}\left(\frac{1}{I} \sum_{i=0}^{I} \tilde{d}_{n}^{J}(i)\right), \quad n \in[0, N-1]
$$

Since the colored noise $\tilde{n}_{n}(i)$ is zero-mean, eq. (8) also holds in the noisy case.

Once we have obtained the estimates $\hat{C}^{J}\left(\rho_{n}\right)$ from (8), the question arises how to find the correct channel coefficients $\hat{C}\left(\rho_{n}\right)$.

\subsubsection{Minimum Impulse Length (MIL) approach}

If the channel order $q$ is known a priori, we will find only one estimate $\hat{c}(k)$ out of $J^{N}$ possibilities which is not longer than $q+1$. Hence, with (8) MIL searches for the shortest impulse response over all possible vectors $\hat{\mathbf{C}}_{1}:=\left[\lambda_{0}\left[\hat{C}^{J}\left(\rho_{0}\right)\right]^{1 / J}, \ldots, \lambda_{N-1}\left[\hat{C}^{J}\left(\rho_{N-1}\right)\right]^{1 / J}\right]^{T}$, where $\lambda_{n} \in\left\{e^{j \frac{2 \pi}{J} m}\right\}_{m=0}^{J-1}$ is a scalar ambiguity corresponding to the $J$ th root, $\forall n$.

After calculating the time domain vectors $\hat{\mathbf{c}}_{1}=\mathbf{F}_{N}^{\mathcal{H}} \hat{\mathbf{C}}_{1}$,

\footnotetext{
${ }^{2}$ For BPSK (Binary Phase Shift Keying), QPSK (Quadrature Phase Shift Keying), and 64-QAM (Quadrature Amplitude Keying) $M=2,4$, and 64 , respectively.
}

where $\mathbf{F}_{N}$ denotes the $N \times N$ FFT matrix

$$
\mathbf{F}_{N}=\frac{1}{\sqrt{N}}\left[\begin{array}{cccc}
1 & 1 & \cdots & 1 \\
1 & e^{-j 2 \pi / N} & \cdots & e^{-j 2 \pi(N-1) / N} \\
\vdots & \vdots & \ddots & \vdots \\
1 & e^{-j 2 \pi(N-1) / N} & \cdots & e^{-j 2 \pi(N-1)^{2} / N}
\end{array}\right]
$$

and ${ }^{\mathcal{H}}$ is the matrix' Hermitian transpose, the correct channel estimate

$$
\hat{\mathbf{c}}=\arg \min _{\hat{\mathbf{c}}_{1}} \sum_{l=q+2}^{N}\left|\hat{c}_{1}(l)\right|^{2}
$$

corresponds to the impulse response with minimum mean power of the coefficients $\hat{c}_{1}(k), k>q+1$. An additional noise reduction according to (6), will further improve the estimation quality of MIL.

However, the main drawback of MIL and MD [6] is an exhaustive search to be performed over $J^{N}$ possible channel coefficient combinations. For QPSK modulated signals $(J=4)$ transmitted over a HIPERLAN/2 or IEEE802.11a channel with $N=52$ active subcarriers this means that $J^{N} \approx 2 \cdot 10^{31}$. Based on the knowledge that the channel impulse response does not violate the cyclic prefix $\left(N_{c p}>q\right)$, MMD [6] reduces this effort to $J^{N_{c p}}$ which is still rather complex, since $N_{c p}=16$ for a HIPERLAN/2 system .

Our new CSC approach is mainly based on the idea of MD and MMD, but dramatically reduces the computational effort so that it will be possible to apply a Finite-Alphabet based blind channel estimator even to high-rate OFDM systems.

\subsubsection{Clustered SubCarrier (CSC) algorithm}

Figure 2 shows the magnitude and phase of a typical
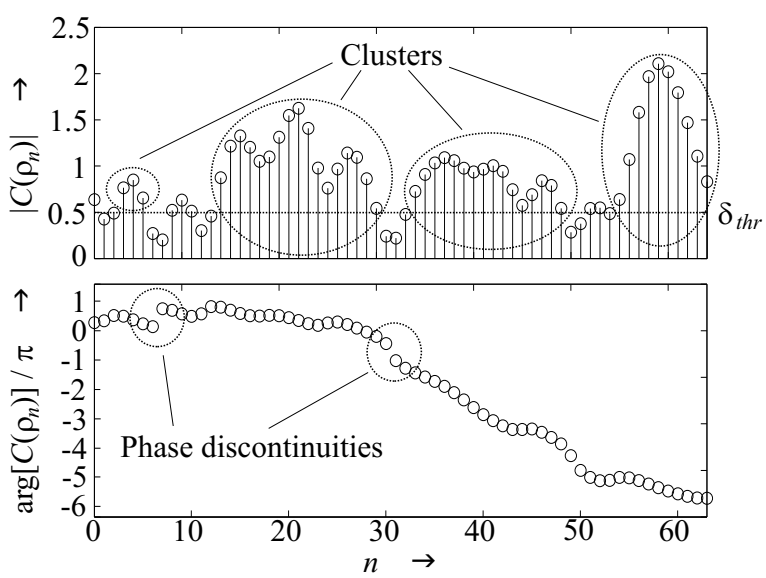

Figure 2: Magnitude and phase of a channel transfer function

HIPERLAN/2 transfer function, where $N=64$. The lower 
subplot depicts a steady phase course, except from the fading subcarriers $n \in\{6,7,30,31\}$, where phase discontinuities are obvious (dotted circles). Hence, it must be possible to track the scalar ambiguity $\lambda_{n}$ of adjacent channel coefficients by choosing their minimum phase distances. This assumption is true as long as no phase discontinuities appear. Figure 3 shows the fading channel coefficients $C\left(\rho_{n}\right)$, $n=6,7$ (a) and $n=30,31$ (b), and some of their neighbours according to Fig. 2 in the complex $z$-plane (black circles). If we compare, for instance, the relation between a) $C\left(\rho_{n}\right), n \in[4,9]$

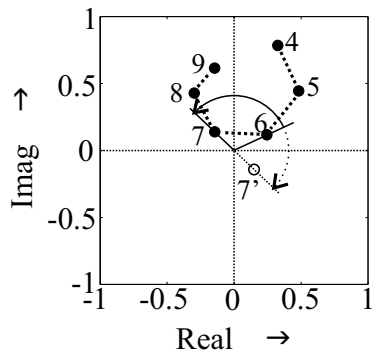

b) $C\left(\rho_{n}\right), n \in[29,33]$

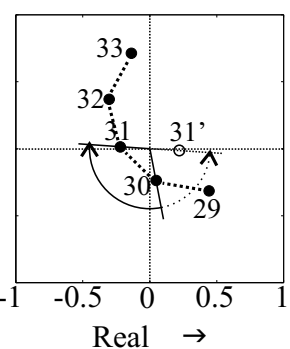

Figure 3: Phase course of fading channel coefficients

$C\left(\rho_{5}\right)$ and $C\left(\rho_{6}\right)$ on the one hand and $C\left(\rho_{6}\right)$ and $C\left(\rho_{7}\right)$ on the other, it is clear that the closer the coefficients are to the origin, the larger their phase differences can be, even if the Euclidean distance remains constant. Under assumption of BPSK modulated signals and starting from $C\left(\rho_{6}\right)$, the choice of the minimum phase difference would lead to the wrong coefficient $C\left(\rho_{7^{\prime}}\right)$ (empty circle) instead of $C\left(\rho_{7}\right)$. Since this error influences all further decisions, a correct estimation of $C\left(\rho_{n}\right)$ will be impossible. Fig. 3b indicates the same behaviour for $29 \leq n \leq 31$. Therefore, we separated the transfer function into different clusters consisting of adjacent strong channel coefficients, whose magnitudes are above a certain threshold $\delta_{t h r}$ (see Fig. 2). Within these clusters, phase discontinuities are very unlikely. Let $\Upsilon$ denote the number of clusters, which is, in general, smaller than 5 . With this, we have dramatically reduced the number of combinations from $J^{N}$ (MD) and $J^{N_{c p}}$ (MMD) to $J^{\Upsilon} \leq 4^{5}=1024$. Moreover, CSC can exploit more subcarriers, since MMD only chooses $N_{c p}$ or $q+1$ elements with largest absolute values. ${ }^{3}$

We will now give an overview of the CSC algorithm according to the MD/MMD scheme presented in [6]:

1. Collect $\hat{C}^{J}\left(\rho_{n}\right)$ from (8) in an $N \times 1$ vektor $\hat{\mathbf{C}}_{J}:=$ $\left[\hat{C}^{J}\left(\rho_{0}\right), \ldots, \hat{C}^{J}\left(\rho_{N-1}\right)\right]^{T}$ and calculate the corresponding reference vector $\hat{\boldsymbol{\beta}}_{J}:=\left[\hat{\beta}_{0}, \ldots, \hat{\beta}_{q J}\right]^{T}=$ $\hat{c}(k) *_{J} \hat{c}(k)$ by IFFT into time domain

$$
\hat{\boldsymbol{\beta}}_{J} \overline{\bar{q} J} \mathbf{Z}_{1}^{\dagger} \hat{\mathbf{C}}_{J}
$$

\footnotetext{
${ }^{3}$ Alternitavely, MMD is focused on $N_{c p}$ or $q+1$ equispaced subcarriers.
}

where $*_{J}$ is the $J$-fold convolution of $c(k)$ with itself and $\mathbf{Z}_{q J+1}:=\sqrt{N} \mathbf{F}_{N}\{:, 1: q J+1\}$ defines an $N \times$ $q J+1$ transform matrix created from the first $q J+1$ columns ${ }^{4}$ of $\mathbf{F}_{N}$ (9). In (11), ${ }^{\dagger}$ denotes the matrix' pseudo-inverse.

2. Calculate $\hat{\mathbf{C}}_{1}$ and separate it into $\Upsilon$ clusters of length $L_{v}, v \in[0, \Upsilon-1]$, where all coefficients that fall below the threshold $\delta_{t h r}$ are omitted due to their phase discontinuity property. The predefinition of the threshold is very important, since clusters might contain phase discontinuities or consist of too little channel coefficients if $\delta_{t h r}$ was set too high or too low, respectively.

3. By exploiting the correlation between adjacent channel coefficients, search their minimum phase distances within each cluster $v$ and track the scalar ambiguity factors

$$
\begin{gathered}
\lambda_{v, \mu}=\arg \min _{\lambda}\left|\hat{C}_{c l}\left(\rho_{v, \mu-1}\right)-\lambda\left[\hat{C}^{J}\left(\rho_{v, \mu}\right)\right]^{1 / J}\right|, \\
\mu \in\left[1, L_{v}-1\right], \quad \lambda_{v, 0}=1,
\end{gathered}
$$

where $\hat{C}_{c l}\left(\rho_{v, \mu}\right)=\lambda_{v, \mu}\left[\hat{C}^{J}\left(\rho_{v, \mu}\right)\right]^{1 / J}$. Collect $\hat{C}_{c l}\left(\rho_{v, \mu}\right)$ in an $L_{v} \times 1$ cluster vector $\hat{\mathbf{C}}_{c l, v}=$ $\left[\hat{C}_{c l}\left(\rho_{v, 0}\right), \ldots, \hat{C}_{c l}\left(\rho_{v, L_{v}-1}\right)\right]^{T}$.

4. Since now every cluster vector $\hat{\mathbf{C}}_{c l, v}$ contains only one scalar ambiguity (instead of $L_{v}$ ), the remaining ambiguities can be resolved by searching over $J^{\Upsilon} \ll J^{N}$ possible vectors $\hat{\mathbf{C}}_{2}=\left[\lambda_{0} \hat{\mathbf{C}}_{c l, 0}, \ldots, \lambda_{\Upsilon-1} \hat{\mathbf{C}}_{c l, \Upsilon-1}\right]^{T}$.

5. Based on the total number of clustered subcarriers $\bar{N}$, calculate the time domain vectors $\hat{\mathbf{c}}_{2}=$ $\overline{\mathbf{Z}}_{q+1} \mathbb{C}$ 2hatvek with $\overline{\mathbf{Z}}_{q+1}:=\sqrt{N} \mathbf{F}_{N}\left\{:_{\bar{N}}, 1: N_{c p}\right\}$. The notation : $\bar{N}$ indicates that $\overline{\mathbf{Z}}_{q+1}$ only contains the rows of $\mathbf{F}_{N}$ belonging to the selected $\bar{N}$ subcarriers. Furthermore, the selection of only $N_{c p}$ columns of $\mathbf{F}_{N}$ corresponds to the noise reduction according to (6).

6. With $\hat{\boldsymbol{\beta}}_{J}$ of (11) channel estimates are finally found by minimizing the Euclidean Distance

$$
\hat{\mathbf{c}}=\arg \min _{\hat{\mathbf{c}}_{2}}\left\|\hat{\boldsymbol{\beta}}_{J}-\hat{\mathbf{c}}_{2} *_{J} \hat{\mathbf{c}}_{2}\right\|
$$

and transforming $\hat{\mathbf{c}}$ into frequency domain.

Since all blind estimators come with an inherent remaining overall scalar ambiguity, this problem can only be solved by the aid of pilot carriers [6]. Furthermore, it must be mentioned that especially with strong noise CSC might not correctly estimate $C\left(\rho_{n}\right)$ when phase discontinuities appear within clusters. In these cases, it is possible to combine

\footnotetext{
${ }^{4}$ According to MATLAB's notation, $\mathbf{B}:=\mathbf{A}(n: m, i: k)$ is a submatrix of $\mathbf{A}$ from $n$th to $m$ th row and from $i$ th to $k$ th column.
} 
a) BERs before Chan. Decod.

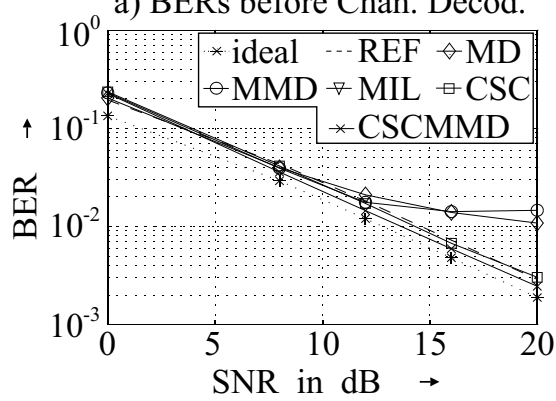

b) BERs after Chan. Decod.

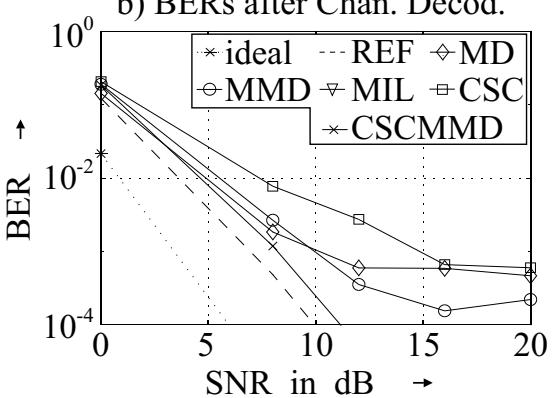

c) Computational Effort

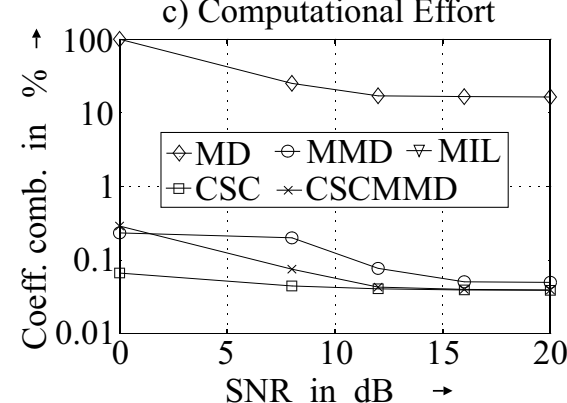

Figure 4: BERs before (a) and after channel decoding (b) for an "ideal", non-blind ("REF") and blind ("MD", "MMD", "MIL", "CSC", "CSCMMD") channel estimation with $N=18, N_{c p}=6, q=4$, and $M=2$. The computational effort of blind channel estimators is shown in subplot (c).

CSC and MMD to the so-called CSCMMD, in order to obtain reliable estimates with still reduced complexity. Furthermore, estimation quality can be improved by an iterative channel estimation, which will be explained in the next section.

\section{Turbo Channel Estimation}

Figure 5 shows the concept of iterative channel estimation within an OFDM receiver. With respect to (3), the

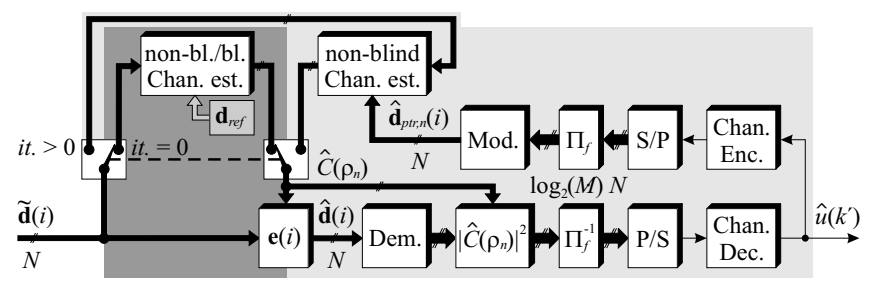

Figure 5: OFDM Turbo Channel Estimation

OFDM symbols $\tilde{\mathbf{d}}(i)$ after CP discarding and FFT transformation are equalized by means of $\mathbf{e}(i)$. Let us first consider the initial step of channel estimation $(i t .=0)$ characterized by the dark grey box and the two switches set to their inner position. According to section 3, e $(i)$ can be based on nonblind or blind channel estimates $\hat{C}\left(\rho_{n}\right)$, where in the nonblind case only the first two OFDM symbols of each burst are utilized for estimation, while blind estimates are based on blocks of $I$ OFDM symbols. After demodulation, each $\log _{2}(M)$ bits have to be multiplied with $\left|\hat{C}\left(\rho_{n}\right)\right|^{2}$ before de-interleaving, if the following channel decoder is based on soft values. Now, the process of iteration (characterized by the light grey box and both switches set to their outer position, it. $>0$ ) starts with re-encoding of the channel decoded bits $\hat{u}\left(k^{\prime}\right)$. Upon S/P conversion, interleaving and modulation, the new pseudo training OFDM symbol $\hat{\mathbf{d}}_{p t r}(i)$ can be utilized by a non-blind channel estimator

$$
\hat{C}\left(\rho_{n}, i\right)=\frac{\tilde{d}_{n}(i)}{\hat{d}_{p t r, n}(i)} .
$$

In order not only to exploit correlations between channel coefficients in frequency domain but also in time domain, $I$ OFDM symbols should be assembled into blocks. On the one hand, this might impair the estimation of time variant channels, but on the other hand the influence of noise can be reduced significantly

$$
\hat{C}\left(\rho_{n}\right)=\frac{1}{I} \sum_{i=0}^{I-1} \hat{C}\left(\rho_{n}, i\right)
$$

In addition, the estimation performance can be improved by tracking the last iteration's channel estimate of each block to the following one [1].

\section{Simulation Results}

In this section, we compare the influence of blind and non-blind channel estimators on the equalization of the received data through MONTE-CARLO simulations. With regard to section 2, 2000 bursts, each consisting of $20 M$-ary modulated OFDM symbols of length $N$, were transmitted over a time invariant ${ }^{5}$ Rayleigh fading channel of order $q$ for different signal-to-noise (SNR) ratios ranging from 0 to $20 \mathrm{~dB}$. In the non-blind case, each burst is preceded by 2 identical training symbols, while in the blind case a burst is separated into blocks of $I=5$ OFDM symbols which only contain information bearing symbols. By comparing the sequences $\hat{b}(k)$ and $b(k)$ on the one hand and $\hat{u}\left(k^{\prime}\right)$ and $u\left(k^{\prime}\right)$ on the other, bit error rates (BERs) were calculated before and after channel decoding, respectively (see Fig. 1), where a half-rate convolutional code with constraint length $L_{c}=5$ was applied.

\footnotetext{
${ }^{5}$ The channel coefficients were changed from burst to burst so that the channel is assumed to be time invariant only over one burst period.
} 
a) BERs before Chan. Decod.

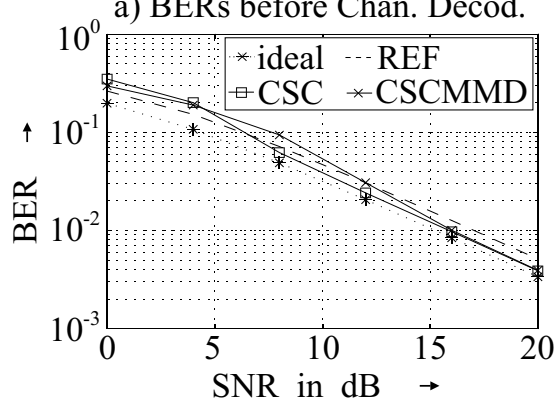

b) BERs after Chan. Decod.

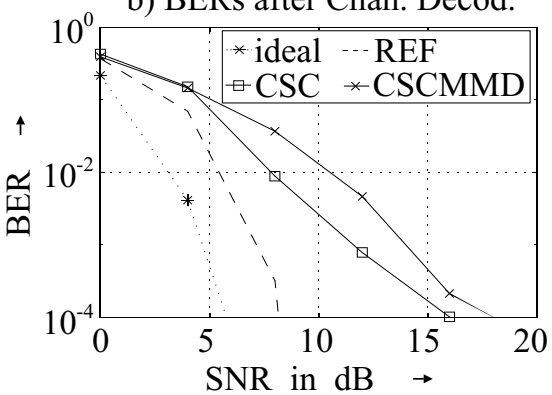

c) Computational Effort

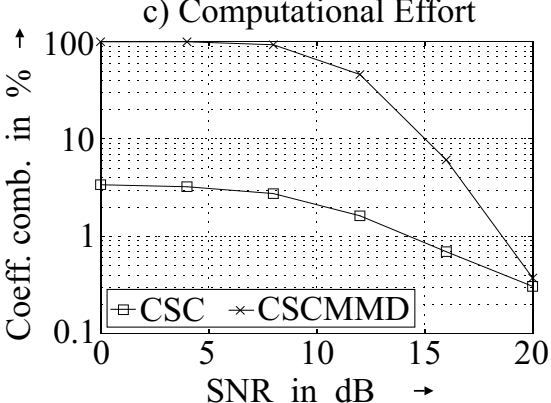

Figure 6: BERs before (a) and after channel decoding (b) with turbo channel estimation after 3 iterations for an initial "ideal", non-blind ("REF") and blind ("CSC", "CSCMMD") estimation, where $N=52, N_{c p}=6, q=2$, and $M=4$ (QPSK). Subplot (c) shows the computational effort of the blind estimators.

Figure 4 shows the BERs before (a) and after channel decoding (b) of BPSK ( $M=2$ ) modulated OFDM symbols with CP length $N_{c p}=6$ transmitted over a Rayleigh fading channel of order $q=4$ with $N=18$ subcarriers for an "ideal", non-blind ("REF"), and blind ("MD", "MMD", "MIL", "CSC", "CSCMMD") channel estimation. From subplot (a) it is obvious that especially for high SNR values CSC outperforms MD and MMD. This is very remarkable, since according to subplot (c) CSC has to check about 500 times less channel coefficient combinations than MD. However, after channel decoding (b) CSC delivers the worst estimation quality of all blind approaches. This effect is caused by remaining burst errors after equalization significantly deteriorating the performance of the channel decoder. On the other hand, the combination of CSC and MMD ("CSCMMD") shows the best performance of all blind approaches, although its computational effort is almost as low as that of CSC (c). There remains only an SNR loss of app. $1 \mathrm{~dB}$ between CSCMMD and the non-blind ("REF") estimator.

Figure 6 depicts the BERs and the computational effort of blind channel estimators for QPSK $(M=4)$ modulated OFDM symbols, where we set $N=52, N_{c p}=6$, and $q=2$. Furthermore, the turbo channel estimation scheme with 3 iterations was applied after an initial "ideal", nonblind ("REF"), and blind ("CSC", "CSCMMD") estimation. The investigation of MD and MIL was impossible due to the enormous number of channel coefficient combinations $\left(4^{52}\right)$ to be checked. From subplot (b) we see that CSC outperforms CSCMMD after channel decoding indicating that the additional MMD approach impairs the estimation quality of CSC. Even if the SNR loss between CSC and REF after channel decoding amounts app. $5 \mathrm{~dB}$ at SNR $=10^{-3}$, we see that it is possible to apply Finite-Alphabet based blind channel estimators to high-rate OFDM systems. Some further investigations will follow in the final paper.

\footnotetext{
${ }^{6}$ Simulation results for MIL will be presented in the final paper. However, for $N=16, N_{c p}=6, q=3$ and $M=2$ MIL outperforms all other blind estimators.
}

\section{Conclusions}

In this paper, we have presented two novel blind channel estimation approaches for OFDM related systems. While MIL shows an excellent estimation performance with high computational effort, our new CSC approach distinguishes through the fact that it enables the application of FiniteAlphabet based blind channel estimators even to high-rate modulated OFDM signals. Furthermore, a turbo channel estimation scheme was introduced which improves the quality of both blind and non-blind estimators by exploiting the capabilities of channel coding.

\section{References}

[1] M. Feuersänger, H. Schmidt, and K. Kammeyer. An Iterative Channel Estimation for a HIPERLAN/2 OFDM System. In Proc. International OFDM Workshop, Hamburg, Germany, September 2000.

[2] J. Heath and G. Giannakis. Exploiting Input Cyclostationarity for Blind Channel Identifikation in OFDM Systems. IEEE Trans. on Signal Processing, 47(3):848-856, March 1999.

[3] R. Klinski, H. Hutzelmann, and R. Knorr. Low Complexity Blind Channel Estimation for OFDM Systems. In Proc. WSES Multiconference on Circuits, Systems, Communications \& Computers (CSCC), Crete, Greece, July 2001.

[4] B. Muquet, M. de Courville, P. Duhamel, and V. Buenac. A Subspace Based Blind and Semi-Blind Channel Identification Method for OFDM Systems. In Proc. IEEE-SP Workshop on Signal Proc. Advances in Wireless Comm., pages 170-173, Annapolis, MD, USA, May 1999.

[5] H. Schmidt, V. Kühn, K. Kammeyer, R. Rückriem, and S. Fechtel. Channel Tracking in Wireless OFDM Systems. In Proc. Multi-Conference on Systemics, Cybernetics and Informatics, Orlando, Florida, USA, July 2001.

[6] S. Zhou and G. Giannakis. Finite-Alphabet based Channel Estimation for OFDM and related Multi-Carrier Systems. IEEE Trans. on Communications, 49, 2001. To appear.

[7] S. Zhou, G. Giannakis, and A. Scaglione. Long Codes for Generalized FH-OFDMA through Unknown Multipath Channels. IEEE Trans. on Communications, 2001. 